Cahiers $d u$ MONDE RUSSE

\section{Cahiers du monde russe}

Russie - Empire russe - Union soviétique et États indépendants

$46 / 3 \mid 2005$

Etrangers en Russie, Russes à l'étranger

\title{
Les famines soviétiques de 1931-1933 et le Holodomor ukrainien
}

Une nouvelle interprétation est-elle possible et quelles en seraient les conséquences?

\section{Andrea Graziosi}

\section{(2) OpenEdition}

\section{Journals}

Édition électronique

URL : https://journals.openedition.org/monderusse/8817

DOI : 10.4000/monderusse. 8817

ISSN : $1777-5388$

Éditeur

Éditions de l'EHESS

\section{Édition imprimée}

Date de publication : 1 juillet 2005

Pagination : 453-472

ISBN : 2-7132-2056-4

ISSN : $1252-6576$

Référence électronique

Andrea Graziosi, «Les famines soviétiques de 1931-1933 et le Holodomor ukrainien », Cahiers du monde russe [En ligne], 46/3 | 2005, mis en ligne le 01 janvier 2007, consulté le 02 septembre 2022. URL : http://journals.openedition.org/monderusse/8817; DOI : https://doi.org/10.4000/monderusse. 8817 
chercher : repérer : avancer

Cet article est disponible en ligne à l'adresse :

http://www.cairn.info/article.php?ID REVUE=CMR\&ID NUMPUBLIE=CMR 463\&ID ARTICLE=CMR 463 0453

\title{
Les famines soviétiques de 1931-1933 et le Holodomor ukrainien
}

\author{
par ANDREA GRAZIOSI
}

\section{Editions de I'EHESS | Cahiers du monde russe}

\author{
$2005 / 3$ - Vol 46 \\ ISSN 1252-6576 | ISBN 2713220564 | pages 453 à 472
}

Pour citer cet article :

—GRAZIOSI A., Les famines soviétiques de 1931-1933 et le Holodomor ukrainien, Cahiers du monde russe 2005/3, Vol 46 , p. $453-472$.

Distribution électronique Cairn pour les Editions de l'EHESS.

(C) Editions de l'EHESS. Tous droits réservés pour tous pays.

La reproduction ou représentation de cet article, notamment par photocopie, n'est autorisée que dans les limites des conditions générales d'utilisation du site ou, le cas échéant, des conditions générales de la licence souscrite par votre établissement. Toute autre reproduction ou représentation, en tout ou partie, sous quelque forme et de quelque manière que ce soit, est interdite sauf accord préalable et écrit de l'éditeur, en dehors des cas prévus par la législation en vigueur en France. Il est précisé que son stockage dans une base de données est également interdit. 


\title{
LES FAMINES SOVIÉTIQUES DE 1931-1933 ET LE HOLODOMOR UKRAINIEN
}

\author{
Une nouvelle interprétation est-elle possible \\ et quelles en seraient les conséquences ? ${ }^{1}$
}

\begin{abstract}
« Tôt ou tard le peuple soviétique vous enverra à la barre des accusés comme traître tant au socialisme qu'à la révolution, principal saboteur, véritable ennemi du peuple, organisateur de la famine... $»^{2}$

F. Raskol'nikov, ambassadeur de l'URSS en Bulgarie, à Stalin, 17 août 1939
\end{abstract}

\section{La découverte}

Entre la fin de 1932 et l'été de 1933, la faim fit en URSS près de sept fois plus de victimes que la Grande Terreur de 1937-1938, en moins de la moitié du temps. Ces mois représentent le pic d'une série de famines qui avaient débuté en 1931, le tournant de la décennie et, en même temps, l'événement le plus important de l'histoire soviétique avant la guerre. Avec ses cinq millions de morts (le chiffre ne tient pas compte des centaines de milliers de gens, peut-être même du million, voire davantage, qui avaient déjà péri au Kazakhstan et ailleurs à partir de 1931), la famine de

1. Une version ukrainienne de cet essai a été publiée dans Ukrä̈ns 'kyj istoryčnyj žurnal. Je remercie les Cahiers du Monde russe qui ont accepté de le publier en français, le rendant plus accessible aux lecteurs occidentaux. Oleg Hlevnjuk et Mark Kramer l'ont lu et m'ont fait bénéficier de leurs critiques. Les hypothèses, les conclusions et les points obscurs sont naturellement de mon fait. Je tiens enfin à remercier Dominique Négrel, ancienne secrétaire de rédaction des Cahiers, qui a bien voulu me traduire, réitérant ce qu'elle avait fait, il y a dix-sept ans, pour les rapports des diplomates italiens sur la famine.

2. C'est moi qui souligne. Raskol'nikov, célèbre héros de la guerre civile, a fait office d'ambassadeur à Sofia de 1934 à 1938 et refusa de retourner à Moscou se faire broyer dans les purges. Sa « lettre ouverte » à Stalin parut dans Novaja Rossija (Paris) le 1er octobre 1939, trois semaines après sa mort à Nice. Cette lettre ainsi que de nombreux autres documents ont été récemment réimprimés dans Reabilitacija: kak eto bylo, fevral' 1956-načalo 80-h godov, Moscou, 2003, p. 420-453. 
1932-1933 fut aussi la plus grave de l'histoire soviétique (tant en 1921-1922 qu'en 1946-1947 il y eut un à deux millions de victimes) sur laquelle elle a laissé une empreinte politique, psychologique et démographique encore visible aujourd'hui. Elle a aussi influencé profondément la vie des pays où résident des communautés immigrées de l'empire russe et d'URSS, et son importance historique et politique est encore forte. Depuis 1987-1988, par exemple, la redécouverte et l'interprétation de la famine ont joué en Ukraine un rôle crucial dans la confrontation entre les partisans de la démocratisation et ceux de l'ancien régime. Le Holodomor (nouveau terme forgé pour définir l'extermination de masse par la faim et son caractère intentionnel $)^{3}$ a occupé le centre du débat politique et culturel, devenant partie intégrante du processus de construction étatique et nationale ; en mai 2003, le parlement ukrainien a déclaré officiellement la famine de 1932-1933 un acte génocidaire perpétré par le régime de Stalin contre le peuple ukrainien.

Pourtant, jusqu'en 1986, lorsque Robert Conquest a publié son livre The Harvest of Sorrow ${ }^{4}$, les historiens avaient quasiment ignoré cet événement extraordinaire. Non que la documentation à ce sujet fît défaut, comme je m'en suis rendu compte en lisant les rapports expédiés par les diplomates italiens à Mussolini qui m'ont montré qu'il avait toujours été possible de savoir. À la faveur des grands mouvements de population du $\mathrm{XX}^{\mathrm{e}}$ siècle - migrations forcées ou encouragées, déplacements, transferts, etc. - et aux traces qu'ils ont laissées - dépêches diplomatiques, comptes rendus de voyageurs, souvenirs des témoins et des victimes nombreuses étaient les voix à parler à qui était capable de les écouter ${ }^{5}$.

3. Le terme a été créé par la fusion des mots holod (en ukrainien, la faim, la famine) et moryty, tuer (par privations), affamer, épuiser ; ce qui met donc l'accent sur l'aspect intentionnel, à la différence du terme plus neutre de holod (golod en russe). Il semble qu'il a été utilisé pour la première fois par l'écrivain Oleksa Musienko dans un rapport à l'organisation du parti de l'Union des écrivains ukrainiens de Kiev (SPU); ce rapport a été publié dans Literaturna Ukraïna, 18/2/1988.

4. R. Conquest, Sanglantes moissons : la collectivisation des terres en URSS, Paris, 1995 (New York, 1986).

5. A. Graziosi, «'Lettres de Kharkov'. La famine en Ukraine et dans le Caucase du Nord à travers les rapports des diplomates italiens, 1932-1934 », Cahiers du Monde russe et soviétique, 30 (1-2), 1989, p. 5-106 ; Id., Lettere da Kharkov. La carestia in Ucraina e nel Caucaso del Nord nei rapporti dei diplomatici italiani, 1932-33, Turin, 1991 ; Commission on the Ukrainian Famine, Investigation of the Ukrainian Famine, 1932-33. Report to Congress, Appendix, Washington, D.C., 1988 ; L. Y. Luciuk, B. S. Kordan, The Foreign Office and the Famine : British Documents on Ukraine and the Great Famine of 1932-1933, Kingston, 1988; D. Zlepko, Der ukrainische Hunger-Holocaust, Sonnenbühl, 1988 (une édition qui laisse à désirer) ; W. W. Isajiw, éd., Famine-genocide in Ukraine, 1932-1933: Western Archives, Testimonies, and New Research, Toronto, 2003 ; V. Kravchenko, J'ai choisi la liberté, Paris, 1947 ; Ukrainian Association of Victims of Russian Communist Terror, The Black Deeds of the Kremlin. A White Book, vol. 2 : The Great Famine in Ukraine in 1932-1933, Detroit, 1955 ; M. Dolot, Execution by Hunger, New York, 1985, etc. Au milieu des années 1960, D. Dalrymple analysa les sources disponibles dans «The Soviet Famine of 1932-34 », Soviet Studies, 3, 1964 et 4, 1965. Il existe actuellement plusieurs bibliographies de la famine et du Holodomor disponibles sur le réseau. Voir par exemple Holodomor 1932-1933. Materialy do bibliografiï, http://www.archives.gov.ua/Sections/Famine/. 
Dans cette perspective, il est stupéfiant de voir le peu qu'on savait et le peu d'intérêt qu'on y portait ${ }^{6}$. Dans le meilleur des cas, des historiens comme Naum Jasny et Alec Nove mentionnaient effectivement une man-made famine (encore envisagée comme s'il s'agissait d'un événement unique), mais sans se préoccuper de l'étudier à fond et en ignorant sa dimension nationale (soit ukrainienne, kazakhe, etc.). Quelques années plus tard, Moshe Lewin reconstitua les mécanismes qui déclenchèrent la famine, mais ne se pencha pas sur celle-ci ${ }^{7}$. Dans le pire des cas, la famine était le prétexte de polémiques navrantes, pour ne pas dire honteuses, dans lesquelles son existence même était contestée ou minimisée. En URSS où les historiens, également après 1956, pouvaient tout au plus évoquer des «difficultés alimentaires », même l'utilisation du mot golod (faim, famine) était interdite. En Ukraine, le terme fut prononcé officiellement pour la première fois par Ščerbyc ${ }^{\prime} \mathrm{kyj}$, premier secrétaire du parti ukrainien, dans le discours qui célébrait le 70e anniversaire de la République en décembre $1987^{\circ}$.

Voilà pourquoi le livre de Conquest, fruit d'un projet lancé par le Harvard Ukrainian Research Institute et codirigé par James Mace', a eu une importance exceptionnelle : il a contraint une profession réticente à se pencher sur un problème fondamental et il l'a fait en mettant soudain en relief le lien entre la famine et la question nationale et en insistant - à juste titre - sur la nécessité de traiter séparément le cas des Kazakhs. Aussi est-il permis de soutenir que l'historiographie relative aux famines et au Holodomor a commencé avec ce livre, même si d'autres chercheurs

6. Ce manque d'attention ne se limitait pas à la famine de 1932-1933. Les études étaient encore dominées, et non sans raison, par l'autorité d'E. H. Carr. Dans ses ouvrages portant sur $1917-$ 1929, il n'avait consacré que quelques pages à la famine de 1921-1922, qui avait pourtant joué un rôle crucial dans l'expérience formative soviétique et dans les développements ultérieurs, sans prendre en considération tant la conduite et le sort des paysans que les répercussions du désastre pour les nationalités impliquées. Nous savons également très peu de choses sur la famine de 1946-1947 malgré l'importance que Hruščev lui a attribuée dans ses mémoires (voir aujourd'hui Vospominanija : vremja, ljudi, vlast', 4 vol., Moscou, 1999). V. F. Zima, Golod v SSSR 1946-1947 godov: proishoždenie i posledstvija, Moscou, 1996 ; O. Veselova et al., Holodomori v Ukraïni 1921-23, 1932-33, 1946-47, Kiev, 2000. K. C. Berkhoff a récemment analysé aussi l' «affamement » de Kiev, organisé par les Allemands en 1941-1942 ; voir son ouvrage Harvest of Despair. Life and Death in Ukraine under Nazi Rule, Cambridge, MA, 2004.

7. N. Jasny, The Socialized Agriculture of the USSR, Stanford, 1949 ; A. Nove, An Economic History of the USSR, Londres, 1969 (3e éd. 1992) ; M. Lewin, «Taking Grain ». Soviet Policies of Agricultural Procurements before the War, Londres, 1974, aujourd'hui dans Id., The Making of the Soviet System : Essays in the Social History of Interwar Russia, New York, 1985 (éd. française : La formation du système soviétique : essais sur l'histoire sociale de la Russie dans l'entre-deux-guerres, Paris, 1987).

8. Sur les circonstances qui conduisirent à son apparition dans le discours, voir le très intéressant essai de S. V. Kul'čyc'kyj, Il tema della carestia nella vita politica e sociale dell'Ucraina alla fine degli anni Ottanta, in G. De Rosa et F. Lomastro, La morte della terra : la grande « carestia » in Ucraina nel 1932-33, Rome, 2004, p. 431-448.

9. Sur James Mace, qui s'est établi ensuite en Ukraine et a disparu récemment, voir la notice biographique de Federigo Argentieri, in G. De Rosa et F. Lomastro, La morte della terra, op. cit., p. 449-453. 
comme Maksudov ou Žores Medvedev étudiaient déjà sérieusement cette question ${ }^{10}$. Ceci est encore plus vrai si on tient compte des polémiques suscitées par ce livre ; grâce à leur niveau, bien supérieur au précédent, le débat, violent lui aussi, sur la « famine » devint partie intégrante du processus par le truchement duquel les historiens commencèrent à prendre conscience des extraordinaires dimensions humaines et intellectuelles de ces événements. Ce processus a été et peut encore être très douloureux parce qu'il intervient après que le jugement historique et la « mémoire collective » d'une époque se sont formés sans que les famines soviétiques en fassent partie. Cela tient à la victoire des tentatives soviétiques d'occulter ce qui s'est passé et à ce que je considère comme une des caractéristiques essentielles du $\mathrm{XX}^{\mathrm{e}}$ siècle européen, dominé par une logique de l'« alignement » qui a provoqué un aveuglement de masse. Il s'est donc agi et il s'agit encore d'introduire les famines soviétiques dans notre représentation du passé, au prix d'une renonciation, parfois cruelle, aux images et aux croyances largement répandues, mais pas véridiques pour autant.

\section{Vers une nouvelle interprétation}

Ensuite survint la révolution archivistique et historiographique de 1991 qui permit l'accumulation accélérée de nouvelles connaissances et fit faire aux polémiques un nouveau saut qualitatif en les transformant, à quelques tristes exceptions près, en controverses scientifiques sérieuses. Un véritable esprit de recherche et un ferme engagement moral, nourris par la conscience de l'immensité de la tragédie qu'on s'efforce de comprendre, animent aujourd'hui les deux camps dans lesquels il est possible - au prix de quelque exagération et de beaucoup de schématisme - de diviser les positions des chercheurs. On peut donc regarder avec satisfaction les années à peine écoulées où les conclusions auxquelles Conquest est parvenu ont été intégrées et en partie dépassées et trouver là un motif d'optimisme.

En schématisant encore un peu plus, les positions de ces deux camps peuvent être synthétisées de la façon suivante (je reprends ici une lettre qui m'a été adressée par Valery Vasil'ev, un jeune et talentueux historien ukrainien) : d'un côté il y a ceux que nous pourrons appeler les chercheurs de type A qui soutiennent la thèse du génocide et voient dans la famine un phénomène organisé artificiellement pour : a) briser les reins des paysans et/ou b) altérer (détruire) le tissu conjonctif vital de la nation ukrainienne qui empêchait la transformation de l'URSS en un empire despotique. De l'autre se trouvent les chercheurs de type B qui, tout en reconnaissant tout à fait la nature criminelle des politiques staliniennes, estiment nécessaire d'étudier la famine comme un «phénomène complexe » dans lequel plusieurs facteurs, de la

10. J. Mace, Communism and the Dilemmas of National Liberation : National Communism in Soviet Ukraine, 1918-1933, Cambridge, MA, 1983 ; S. Maksudov [Babënišev], Poteri naselenija SSSR, Benson, 1989. Le livre de Žores Medvedev, Soviet Agriculture (New York, 1987) contient un chapitre excellent sur la famine dans lequel sont correctement reconstituées ses caractéristiques générales à l'échelle de l'Union soviétique, mais qui ne se préoccupe pas de ses aspects nationaux. Voir aussi B. Krawchenko, Social Change and National Consciousness in Twentieth-Century Ukraine, Edmonton, 1985. 
situation géopolitique à l'effort modernisateur, jouèrent un rôle décisif à côté des intentions et des décisions de Moscou ${ }^{11}$.

Je pense que nous avons aujourd'hui une grande partie des éléments nécessaires à une nouvelle hypothèse interprétative, plus satisfaisante, capable de tenir compte tant du contexte soviétique général que de l'importance de la question nationale ${ }^{12}$. Elle peut être échafaudée à partir des excellents travaux publiés ces dernières années par les historiens ukrainiens, russes et occidentaux, brisant ainsi le mur qui sépare encore, du moins partiellement, leurs efforts.

Dans les prochaines pages, je m'efforcerai d'esquisser les contours de cette interprétation, en me basant sur les recherches de chercheurs éminents comme Danilov, D’Ann Penner et Kondrašin, Davies et Wheatcroft, Ivnickij, Kul'čyc'kyj, Mace, Martin, Meslé et Vallin, Šapoval, Vasil'ev' ${ }^{13}$, et d'Oleg Hlevnjuk dont les travaux sur Stalin et la recherche, bien qu'ils ne soient pas directement centrés sur la famine, nous permettent de la situer dans le contexte politique qui fut le sien ${ }^{14}$.

On espère ainsi non seulement faire progresser la compréhension de la « Grande famine» (terme collectif pour désigner les famines de 1931-1933 dans leur ensemble), mais aussi stimuler un débat qui contribue à abattre le mur, encore haut et solide, qui sépare ses historiens de ceux qui étudient le $\mathrm{XX}^{\mathrm{e}}$ siècle européen, un siècle qu'il est tout simplement impossible de comprendre et de juger complètement sans que ces événements y soient définitivement intégrés.

11. Étant donné la schématisation extrême de cette classification, il me paraît difficile d'attribuer à l'un ou l'autre camp tel point de vue ou tel auteur. Mais même les meilleures études comme celles qui sont citées dans la note 13 pourraient souvent, sans grande difficulté, être subdivisées en A et B.

12. En 1996, dans The Great Soviet Peasant War, 1918-1933, Cambridge, MA., j’avais déjà cherché à rassembler une telle hypothèse, mais d'une façon que je tiens aujourd'hui pour inadéquate et au moins en partie fausse.

13. V. P. Danilov et al., éd., Tragedija sovetskoj derevni, vol. 3 : 1930-1933, Moscou, 2001 (cité infra : Tragedija) ; R. W. Davies, O. Khlevniuk et al., éds., The Stalin-Kaganovich Correspondence, 1931-1936, New Haven, CT, 2003 (Id., Stalin i Kaganovič : Perepiska 1931-1936 gg., Moscou, 2001) ; R. W. Davies, S. G. Wheatcroft, The Years of Hunger: Soviet Agriculture, 1931-1933, New York, 2004 ; N. A. Ivnickij, Kollektivizacija i raskulačivanie, Moscou, 1996 ; Id., Repressivnaja politika sovetskoj vlasti v derevne, 1928-1933, Moscou, 2000 ; V. Kondrašin, D. Penner, Golod: 1932-1933 v sovetskoj derevne (na materiale Povolž ja, Dona i Kubani), Samara-Penza, 2002 ; S. V. Kul’čyc’kyj, éd., Holod 1932-1933 rokiv na Ukraïni: očyma istorykiv, movoju dokumentiv, Kiev, 1990 ; Id., éd., Holodomor 1932-1933 rr. V Ukraïni: pryčyny i naslidky, Kiev, 1993 ; Id., Kolektyvizacija i holod na Ukraïni, 1929-1933, Kiev, 1993 ; Id., Ukraïna miž dvoma viynamy (1921-1939 rr.), Kiev, 1999 ; V. M. Litvin, éd., Holod 1932-33 rokiv v Ukraïni: pryčyny ta naslidky, Kiev, 2003 ; T. Martin, The Affirmative Action Empire: Nations and Nationalism in the Soviet Union, 1923-1939, Ithaca, NY, 2001 ; F. Meslé, J. Vallin, Mortalité et causes de décès en Ukraine au XX $X^{\mathrm{e}}$ siècle, Paris, 2003 ; Ju. Šapoval, V. Vasil’ev, Komandiry velykoho holodu: poïzdky V. Molotova i L. Kahanovyča v Ukraïnu ta na Pivničnyj Kavkaz, 1932-33 rr., Kiev, 2001. Les recherches que Timothy Snyder est en train de diriger sur les relations polono-ukraino-soviétiques sont également d'un grand intérêt. Voir par exemple «A National Question Crosses a Systemic Border: The Polish-Soviet Context for Ukraine, 1926-1935 », intervention au congrès de la Società Italiana per lo Studio della Storia Contemporanea (Sissco), Bolzano-Bozen, septembre 2004.

14. O. Khlevniouk [Hlevnjuk], Le cercle du Kremlin. Stalin et le Bureau politique dans les années 30, Paris, 2005 (et, plus complet : Politbjuro. Mehanizmy političeskoj vlasti v 1930-e gody, Moscou, 1996); O. Khlevniuk [Hlevnjuk], The History of he GULAG, New Haven, 2004. 
Pour formuler cette nouvelle interprétation, il convient tout d'abord de définir avec précision son objet. Comme cela devrait être clair désormais, nous avons affaire à ce qu'il serait plus correct d'appeler, au niveau soviétique, les famines de 1931-1933 qui eurent naturellement des causes communes et un arrière-plan comparable, mais qui comprennent au moins deux phénomènes dont l'importance est aussi grande que leurs différences : la famine kazakhe avec les épidémies de 1931-1933 et le Holodomor ukrainien et du Kouban (une région du Caucase du Nord appartenant à la République russe mais alors peuplée principalement d'Ukrainiens) de fin 1932 au début 1933.

Bien des incompréhensions passées ont leur racine dans la confusion entre ces deux tragédies nationales et le phénomène plus général qui lui servit d'arrière-plan. Dans un certain sens, c'est comme si les historiens du nazisme confondaient la répression nazie en général avec certains cas spécifiques, cruciaux, comme l'extermination des prisonniers de guerre soviétiques, ou celle des Polonais et des Tziganes, sans parler de l'Holocauste, un phénomène exceptionnel qui ne peut être expliqué comme un simple aspect ou un élément des exterminations nazies, bien qu'il en ait fait partie évidemment. En somme, tant la répression nazie en général que ces tragédies « spécifiques » ont existé et il faut, comme cela se passe effectivement, tenir compte des deux niveaux, en étudiant tant les phénomènes en euxmêmes que leurs liens et le cadre général qu'ils composent.

Dans le cas soviétique aussi, il serait donc opportun de faire une distinction nette entre le phénomène général et ses manifestations dans les républiques et dans les régions du pays. Mais un grand nombre de défenseurs de la position A se réfèrent en fait au Holodomor, tandis que de nombreux partisans de la position B raisonnent à l'échelle soviétique. Si nous faisions vraiment cette distinction, nous finirions par découvrir que chez beaucoup d'entre eux, mais certes pas chez tous, les deux points de vue sont dans le vrai, dans leur champ de recherche respectif.

Le deuxième pas qu'il convient de faire, c'est d'opérer une distinction analytique ultérieure : il faut en fait séparer les famines «spontanées » de 1931-1932 — les guillemets sont nécessaires car elles ont été naturellement les conséquences directes des choix de 1928-1929, même si elles furent non programmées et imprévues - de la famine postérieure à septembre 1932 provoquée avant tout par des décisions humaines directement liées à elle (les événements du Kazakhstan se déroulèrent selon un parcours en grande partie différent et je me bornerai donc à les traiter uniquement lorsque ce sera nécessaire et par conséquent au passage, en renvoyant aux travaux les plus récents sur ce sujet qui, à mon avis, les ont finalement retracés de façon satisfaisante) ${ }^{15}$.

15. K. Aldažumanov et al., Nasil'stvennaja kollektivizacija i golod v Kazahstane v 1931-1933 gg., Alma-Ata, 1998 ; I. Ohayon, « 'Du nomadisme au socialisme'. Sédentarisation, collectivisation et acculturation des Kazakhs en URSS (1928-1945) », Thèse de doctorat, Institut national des langues et civilisations orientales, Paris, 2003; N. Pianciola, «Famine in the Steppe. The Collectivization of Agriculture and the Kazakh Herdsmen, 1928-1934 », Cahiers du Monde russe, 45(1-2 ), 2004, p. 137-192. 
Le troisième pas à faire consiste à réunir et à combiner les éléments les plus convaincants des hypothèses $\mathrm{A}$ et $\mathrm{B}$, en laissant tomber leurs parties les moins satisfaisantes.

Les études de type A ont raison, par exemple, d'attirer notre attention sur la question nationale. Quiconque étudie l'Union soviétique devrait être conscient de son importance, comme le furent tant Lenin que Stalin (après tout le premier décida de ne pas appeler Russie le nouvel État, et le second, qui était au début opposé à ce choix, ne le changea pas au cours des années suivantes). Il faudrait ensuite avoir conscience de la primauté de l'Ukraine dans ce domaine. On a, à juste titre, fait remarquer que l'Ukraine a joué, après 1917 , le rôle dévolu à la Pologne dans l'empire tsariste : à la fin de 1919, Lenin amorça le tournant vers les politiques d'indigénisation (korenizacija) ${ }^{16}$, jusqu'alors apanage des formations nationalistes plus radicales, suite à ses réflexions sur les raisons de la défaite bolchevique en Ukraine au printemps et à l'été $1919^{17}$, et Stalin donna à la korenizacija une nouvelle direction à la fin de 1932 à cause de la crise ukrainienne. Mais en Ukraine, du moins jusqu'en 1933, la question nationale était la question paysanne, et c'est ce que pensaient à juste titre aussi bien Lenin que Stalin.

Pour des motifs que nous verrons, l'hypothèse A au contraire n'est pas fondée quand elle soutient que la « famine », y compris la famine à l'échelle de l'Union, a été organisée («planifiée ») même avant l'automne 1932 pour résoudre le problème national et/ou paysan ukrainien.

Les chercheurs du groupe B proposent une reconstitution précieuse et détaillée des causes et du contexte général de la famine au niveau soviétique, dans toute sa complexité, et ils sont ainsi capables d'apporter une critique convaincante de l'hypothèse $\mathrm{A}$, du moins dans ses versions les plus simplistes. Et pourtant ils semblent incapables de comprendre pleinement le facteur national, et donc de lui faire place, c'est-à-dire de « descendre » du niveau de l'Union à celui des républiques. Ces chercheurs, en outre, ne paraissent pas toujours en mesure de voir que Stalin, même lorsqu'il n'en était pas l'initiateur, était toujours prêt à profiter d'événements « spontanés », en imprimant une nouvelle direction et une réorientation à leur cours. Le parallèle le plus évident est celui de l'assassinat de Kirov que Stalin très probablement n'avait pas organisé, mais qu'il sut certes exploiter de façon « créative ».

Il est donc possible d'utiliser les bonnes études de type B sur le développement de la crise en URSS, en ajoutant cependant qu'au niveau de l'Union aussi Stalin

16. En 1923, une fois l'URSS organisée en une fédération de républiques basées sur les nationalités titulaires, le parti adopta officiellement un ensemble de mesures pour promouvoir le développement des nationalités « arriérées », en garantissant leurs privilèges et leurs droits particuliers dans leur territoire. Rapidement de telles mesures prirent le nom collectif de korenizacija (indigénisation), dérivé du mot koren', racine, qui montrait bien que leur but était l'enracinement du régime dans les zones non russes. Voir T. Martin, The Affirmative Action Empire..., op. cit.

17. Richard Pipes dans The Unknown Lenin (New Haven, CT, 1996, p. 76-77) a publié les ébauches secrètes des thèses sur la « politique en Ukraine » rédigées par Lenin au moment de la reconquête de la république en novembre 1919. Entre autres choses, Lenin demandait « la plus grande attention à l'égard des traditions nationales, le strict respect de l'égalité de la langue et de la culture ukrainiennes », etc., et ordonnait néanmoins de «traiter les Juifs et les habitants des centres urbains (dans une très grande mesure non ukrainiens) avec une poigne de fer ». 
décida à un certain moment d'utiliser la famine pour briser la résistance des paysans à la collectivisation. Pour de multiples raisons, cette opposition avait été en général plus forte dans les secteurs non russes, où les événements commencèrent rapidement à se développer en suivant des parcours autonomes. En reconstituant ces parcours, il devient possible de percer le secret qui a enveloppé les événements de 1932-1933 dès leur apparition, un secret que pourtant (comme semble le suggérer la lettre de Raskol'nikov) l'élite bolchevique connaissait naturellement.

\section{La spécificité ukrainienne et l'utilisation politique de la famine}

Qu'est-il donc possible de dire ? En 1931-1933 des centaines de milliers de personnes moururent de faim dans toute l'Union soviétique. Au Kazakhstan et en Ukraine, dans le Caucase du Nord et dans la Basse- et la Moyenne-Volga, la situation était pourtant incomparablement plus grave. À l'exception du Kazakhstan (et sans compter la Sibérie occidentale), il s'agissait des centres les plus importants de production céréalière du pays, où - à partir de 1927 - le conflit entre l'État et les paysans pour la récolte avait été le plus aigu. En outre, au moins à partir de 19181919, la guerre entre le régime et les paysans (ou les nomades) avait pris des traits particulièrement brutaux en raison de la présence de facteurs nationaux et/ou religieux qui en décuplaient l'intensité (en Moyenne- et en Basse-Volga, il fallait au contraire compter, outre la forte présence de colons allemands, avec les grandes traditions du mouvement paysan russe, d'inspiration socialiste-révolutionnaire).

Encore une fois à l'exception du Kazakhstan, les causes du phénomène étaient partout similaires : l'impact dévastateur, sur le plan humain comme sur celui de la production, de la dékoulakisation qui se termina par un pogrom dirigé par l'État contre l'élite paysanne ; la collectivisation forcée qui avait incité les paysans à détruire une grande partie de leurs biens ${ }^{18}$; le mauvais fonctionnement et la misère des kolkhozes ; des vagues de réquisitions impitoyables et répétées qui tiraient leur origine de l'industrialisation en crise, de l'urbanisation incontrôlée et de la croissance de la dette extérieure à laquelle on pouvait faire front seulement en exportant des matières premières ; la résistance des paysans qui n'acceptaient pas ce qu'ils définirent aussitôt comme un « second servage » et qui travaillaient de moins en moins, soit par refus du nouveau système, soit en raison de leur affaiblissement physique dû au manque de nourriture ; les mauvaises conditions météorologiques de 1932. La famine, qui avait commencé à frapper ici et là en 1931 (pourtant au Kazakhstan les nomades mouraient déjà en masse) et formé des poches importantes au printemps 1932, apparut donc comme la conséquence imprévue, non programmée, des politiques d'inspiration idéologique,

18. Dans un rapport à l'OGPU sur les réquisitions de céréales de mai 1929, il est déjà fait mention de protestations paysannes déchaînées par la confiscation du pain et d'autres denrées de première nécessité dans les villages qui n'avaient pas rempli le plan de livraison. La faim fut donc utilisée par le régime qui reprenait également dans ce cas les pratiques de la guerre civile pour punir et « éduquer » les paysans depuis les débuts de la collectivisation. Voir N. Werth et G. Moullec, Rapports secrets soviétiques, Paris, 1994,p. 112. 
c'est-à-dire marxiste, sur lesquelles la direction bolchevique avait misé pour déraciner la production privée des marchandises. Certes, sur la base des résultats du communisme de guerre (dont beaucoup de principes furent repris en 1928-1929) en 19201921, il n'aurait pas été difficile en 1928-1929 de prévoir ce qui se produisit ensuite en 1931-1933. Mais si on analyse les origines et l'évolution de la famine avant l'automne 1932 à l'échelle de l'URSS, comme l'ont fait récemment Robert Davies et Stephen Wheatcroft par exemple ${ }^{19}$, il semble très difficile de soutenir que la famine fut l'effet attendu de ces politiques, comme on le fait parfois dans les hypothèses présentant la grande famine comme un événement voulu en sorte de briser la résistance paysanne ou pour perpétrer un génocide anti-ukrainien planifié à Moscou (quand ce n'est pas carrément, et injustement, par les « Russes »).

Toutefois, l'intensité, la progression et les conséquences du phénomène que les nouveaux documents et études nous permettent d'analyser varièrent indiscutablement, et de façon importante, dans les différentes régions et républiques. Sur les 5 à 6 millions de victimes en 1932-1933 (aujourd'hui les démographes imputent à 1930-1931 une partie des morts attribués au préalable aux deux années suivantes), 3,5 à 3,8 millions moururent en Ukraine ; 1,3 à 1,5 million au Kazakhstan (où la mortalité atteignit son point culminant, en exterminant 33 à $38 \%$ des Kazakhs et 8 à $9 \%$ des Européens); enfin, plusieurs centaines de milliers dans le Caucase du Nord et, dans une moindre mesure, en Moyenne- et en Basse-Volga où le secteur le plus frappé coïncidait largement avec la République autonome allemande (définitivement dissoute en 1941) $)^{20}$.

Le taux de mortalité annuel pour mille habitants dans les campagnes, égal à 100 en 1926, sauta à 188,1 en 1933 dans l'ensemble du pays. Mais, en cette même année, il était égal à 138,2 dans la République russe (qui comprenait pourtant alors aussi bien le Kazakhstan que le Caucase du Nord), et à 367,7, autrement dit presque le triple, en Ukraine. Là, l'espérance de vie à la naissance tomba en 1933 de 42,9 ans pour les hommes et 46,3 pour les femmes de 1926, à 7,3 et 10,9 ans respectivement (elle aurait été de 13,6 ans pour les hommes et 36,3 pour les femmes en la terrible année 1941 qui s'avère ainsi cependant moins terrible que 1933). Toujours en Ukraine, les 1153000 naissances attestées en moyenne entre 19261929, régressèrent à 782000 en 1932 et tombèrent à 470000 en $1933^{21}$.

Ces écarts reflètent le cours différent suivi par la famine, cours dont étaient largement responsables les décisions politiques prises à Moscou qui, à partir de

19. R. W. Davies, S. G. Wheatcroft, The Years of Hunger ..., op. cit.

20. L'incertitude des données ukrainiennes et surtout des données kazakhes provient en premier lieu de la difficulté d'évaluer l'impact précis des exodes provoqués par la famine. Un grand nombre de fuyards furent refoulés et renvoyés mourir chez eux, d'autres périrent dans les gares des villes qu'ils avaient réussi à atteindre, d'autres encore réussirent à se sauver en Russie, en Transcaucasie ou en Chine.

21. S. Maksudov, Poteri naselenija SSSR, op. cit.; S. V. Kul’̌yc’kyj, éd., Holomor 19321933..., op. cit. ; R. W. Davies, S. G. Wheatcroft, The Years of Hunger ..., op. cit. ; F. Meslé, J. Vallin, Mortalité et causes de décès..., op. cit. ; E. M. Andreev, L. E. Darskij, T. L. Har'kova, Demografičeskaja istorija Rossijskoj federacii, 1927-1959, Moscou, 1998 ; Ju. A. Poljakov, éd., Naselenie Rossii v XX veke, vol. 1 : 1900-1939 gg., Moscou, 2000. 
l'automne 1932, donnèrent à celle-ci, dans des républiques et des régions déterminées, ce caractère «planifié », parfois projeté de façon erronée également sur la période précédente.

En Ukraine comme dans bien d'autres secteurs du pays, au printemps 1932, des fonctionnaires locaux, des instituteurs de village et des dirigeants de la république observèrent l'extension de la famine et le début d'un exode rural de masse ${ }^{22}$. Stalin, sous la pression du parti ukrainien qui réclamait une réduction des plans de réquisition, admit au début de juin que, au moins dans les zones où la situation était la plus difficile, cette mesure était nécessaire, même par «sens de la justice ». De telles réductions devaient cependant être modestes et locales, car comme Molotov l'aurait rapidement déclaré officiellement, "même si nous sommes confrontés aujourd'hui au spectre de la famine, surtout dans les zones productrices de céréales, [...] les plans de ramassage doivent à tout prix être respectés» ${ }^{23}$, conclusion à laquelle poussait la nécessité d'éviter la répétition à grande échelle des grèves et des mouvements urbains du printemps, et d'honorer les traites allemandes venant à échéance entre la fin de l'année et le début de 1933.

Pourtant, toujours en juin 1932, bien avant que les nationalistes ukrainiens aient seulement envisagé une telle hypothèse, Stalin était en train d'élaborer ce que Terry Martin a appelé à juste titre son « interprétation nationale » de la famine ${ }^{24}$. Dans un premier temps, il donna, en privé, libre cours à sa rage contre les dirigeants de la république qu'il tenait pour responsables d'une situation à laquelle ils étaient incapables de faire face avec la fermeté nécessaire. Cependant, quelques semaines plus tard, entre juillet et août, après une conférence du parti ukrainien, implicitement polémique avec Moscou et sur la base des rapports de l'OGPU qui accusaient les communistes ukrainiens d'être contaminés par le nationalisme, Stalin mettait au point une nouvelle analyse de la situation et de ses causes ${ }^{25}$.

Il n'est pas impossible que ce qui fut l'ultime occasion de désaccord au sein du Politbjuro avant la mort de Stalin dont on trouve trace dans les dossiers ait aussi joué un rôle. À la réunion du 2 août 1932, quelqu'un, - probablement Petrovskij, alors président de la République ukrainienne - , contesta l'ébauche préparée par Stalin

22. La tragédie de 1921-1922, elle aussi, fut annoncée par les famines locales du printemps précédent, celui de 1920. Voir A. Graziosi, «Stato e contadini nelle Repubbliche sovietiche attraverso i rapporti della polizia politica, 1918-1922 », Rivista storica italiana, II, 1998, p. 463528 (dans lequel, toutefois, je cite de façon erronée des données anciennes surestimant la mortalité due à cette famine); B. Patenaude, The Big Show in Bololand: The American Relief Expedition to Soviet Russia in the Famine of 1921, Stanford, 2002.

23. Cité dans N. A. Ivnickij, «Golod 1932-1933 godov: kto vinovat », in Golod 1932-1933 godov, Moscou, 1995, p. 59.

24. La meilleure reconstitution des origines de l' « interprétation nationale » donnée à la famine par Stalin se trouve dans T. Martin, The Affirmative Action Empire..., op. cit. Cependant, Mace aussi était arrivé précédemment à des conclusions très semblables. Il avait également l'intuition que quelque chose de décisif pour les développements ultérieurs s'était produit en juillet 1932 .

25. Le 5 août, par exemple, l'OGPU rapportait que des fractions du parti communiste ukrainien et des nationaux-socialistes ukrainiens étaient « aux ordres du deuxième bureau de l'état-major polonais », in Tragedija, p. 420-422, 433. 
(ce dernier était en vacances et ne participait donc pas à la séance); il s'agissait de l'ébauche de ce qui allait devenir le décret draconien, tristement célèbre, du 7 août sur la défense de la propriété d'État contre le vol dans les champs ${ }^{26}$. Tout de suite après, le 11 août, malgré la récente signature du pacte soviéto-polonais de non-agression $^{27}$, dans une lettre cruciale à Kaganovič, Stalin écrivit que l'Ukraine était désormais la «principale question » (souligné par Stalin), que le parti, l'État et même les organes de la police politique de la république étaient infestés d'agents nationalistes et d'espions polonais, qu'on courait le risque « de perdre l'Ukraine », une Ukraine qu'il fallait transformer au contraire en une « forteresse bolchevique $»^{28}$.

Cette interprétation, développée à la base de l'expérience ukrainienne, fut ensuite étendue aux Cosaques qui avaient été classés comme ennemis du régime dès 1919 lorsqu'ils avaient été frappés par la « décosaquisation » ${ }^{29}$, aux Allemands de la Volga et, en termes moins brutaux toutefois, aux Biélorusses. La crise poussa par conséquent Stalin à appliquer son modèle déjà très élaboré de répression préventive, catégorielle et donc collective (qui avait déjà atteint son premier point culminant avec la dékoulakisation) à de nombreux groupes nationaux et socionationaux lesquels, à son avis, représentaient une menace pour le régime. Comme les événements le prouveraient, l'Ukraine et les Ukrainiens continuaient pourtant à être en tête de ses préoccupations.

Lorsque, comme il fallait s'y attendre, les collectes furent insuffisantes dans les régions traditionnellement productrices de céréales, Molotov, Kaganovič et Postyšev furent envoyés respectivement en Ukraine, dans le Caucase du Nord et dans le bassin de la Volga pour redresser la situation. La décision d'utiliser la famine, en en amplifiant artificiellement l'étendue pour donner une leçon aux paysans qui refusaient le «nouveau servage ${ }^{30}$, fut donc prise en automne au

26. Kaganovič évoqua cette opposition sans mentionner directement Petrovskij, dans une lettre à Stalin qui n'a peut-être pas été envoyée : « Nous venons de nous réunir pour discuter du projet de décret qui se compose de trois parties dans l'esprit de vos indications. Contre la troisième partie est intervenu hier ..., qui pourtant n'était pas là aujourd'hui, étant parti. Des doutes et même des objections à propos de la seconde et de la troisième partie ont aussi été formulés par ..., mais en fin de compte nous avons adopté le texte tel qu'il avait été conçu. » La seconde partie condamnait les responsables de vol de la propriété kolkhozienne (essentiellement le blé) à la peine de mort, ou à 5 à 10 ans de travaux forcés en présence de circonstances atténuantes. La troisième partie punissait celui qui incitait les paysans à quitter les kolkhozes de 5 à 10 ans de travaux forcés. In Stalin i Kaganovič : Perepiska ..., op. cit.

27. Le pacte fut signé le 25 juillet 1932. Dans son étude «A National Question Crosses a Systemic Border...», Snyder soutient de façon convaincante que même si Moscou, après le coup d'État de Piłsudski de 1926, se sentit l'objet d'une possible attaque, Varsovie devint, après 1930, toujours plus favorable au maintien du statu quo. Il est donc probable, comme le suggère Snyder, qu'une fois la menace polonaise éliminée avec le pacte pendant l'été 1932, Stalin se soit senti libre d'en exploiter les résidus pour liquider les ennemis potentiels et consolider sa position.

28. Stalin i Kaganovič : Perepiska..., op. cit., p. 273-274 et Y. Cohen, « Des lettres comme action », Cahiers du Monde russe, 38(3), 1997, p. 307-346.

29. P. Holquist, «'Conduct Merciless Mass Terror.' Decossackization on the Don, 1919 », Cahiers du Monde russe, 38 (1-2 ), 1997, p. 127-162.

30. S. Fitzpatrick, Stalin's Peasants: Resistance and Survival in the Russian Village after Collectivization, New York, 1994 ; M. A. Beznin, T. M. Dimoni, « Povinnosti rossijskih kolhoznikov v 1930-1960-e gody », Otečestvennaja istorija, 2, 2002. 
moment où la crise provoquée par le premier plan quinquennal atteignit son point culminant et où la femme de Stalin se suicida. La punition était tragiquement simple, presque pavlovienne ; au fond elle n'était pas étrangère aux vieux clichés socialistes qui acquéraient ainsi une tout autre signification : qui ne travaille pas, c'est-à-dire qui n'accepte pas le système kolkhozien, ne mange pas. Stalin se référa à la méthode qu'il avait imaginée dans sa fameuse correspondance de 1933 avec Šolohov. Les « cultivateurs de blé estimés » du Don, dont le célèbre écrivain avait déploré le sort avec Stalin, avaient engagé « une guerre 'secrète' contre le pouvoir soviétique, une guerre dans laquelle - écrivait Stalin prenant le contre-pied de la réalité - ils avaient utilisé la faim comme une arme », et dont ils payaient maintenant les conséquences (autrement dit avec la famine qui sévissait, d'après la conclusion implicite que Stalin laissait deviner) ${ }^{31}$.

Moscou refusa de porter secours à la grande majorité des régions les plus frappées jusqu'au printemps de 1933 (même les paysans du Don ne reçurent rien avant mai). Alors que le commissaire aux Affaires étrangères Litvinov niait officiellement l'existence même de la famine dans ses réponses aux questions des diplomates et de la presse étrangère, l'État en effet « luttait avec férocité » (selon les propos de Kaganovič) pour mener à terme les plans de réquisition dans les régions en question.

Là où la question nationale compliquait la «question paysanne », en la rendant plus aiguë et donc plus dangereuse - rappelons que Stalin avait explicitement lié les deux questions dans ses écrits sur le nationalisme et que le leadership soviétique avait vu cette hypothèse se confirmer par les grandes révoltes sociales et nationales des campagnes ukrainiennes en 1919, qui s'étaient répétées ensuite, bien qu'à une échelle mineure, au début de $1930^{32}$ - le recours à la faim fut plus impitoyable et la leçon beaucoup plus cruelle. D'après les données démographiques, en Ukraine aussi la mortalité était étroitement liée à la résidence, urbaine ou rurale, et non à la nationalité des victimes. En d'autres termes, celui qui vivait à la campagne souffrit bien plus que celui qui résidait en ville, indépendamment de ses « origines ethniques ». Mais on ne peut oublier que, comme tout le monde le savait, malgré l'urbanisation accompagnée de l'ukrainisation de la décennie précédente, les villages restaient en majorité ukrainiens, alors que les villes avaient en grande partie conservé leur caractère « étranger », c'est-à-dire russe, juif et - toujours dans une moindre mesure - polonais ${ }^{33}$. Par

31. Pour cet échange révélé par Hruščev en 1963, voir aujourd'hui Pisatel'i i vožd': perepiska M. A. Šolohova s I. V. Stalinym, Moscou, 1997.

32. A. Graziosi, Bol’̌̌seviki i krest’jane na Ukraine, 1918-1919 gody, Moscou, 1997 ; Id., «Collectivisation, révoltes paysannes et politiques gouvernementales à travers les rapports du GPU d'Ukraine de février-mars 1930 », Cahiers du Monde russe, 35 (3), 1994, p. 437-632; L. Viola, Peasant Rebels under Stalin : Collectivization and the Culture of Peasant Resistance, New York, 1996 ; V.P. Danilov, A. Berelowitch, éds., Sovetskaja derevnja glazami VČKOGPU-NKVD, vol. 3 : 1930-1934, t. 1 : 1930-1931, Moscou, 2003.

33. Stalin ne se préoccupa jamais des « éclats qui volent quand on taille le bois », pour utiliser une de ses expression favorites. Et il fut peut-être le plus grand interprète et exécutant de l'école «statistique » de répression, prête à éliminer des catégories entières pour garantir la «solution» de problèmes réels, ou même seulement potentiels ou imaginaires. Voir A. Graziosi, O. Chlevnjuk, T. Martin, « Il grande terrore », Storica, 18, 2000, p. 7-62. 
conséquent, en Ukraine aussi, les campagnes furent l'objectif des mesures visant à briser les reins des paysans, mais on avait pleinement conscience que ces campagnes représentaient l'épine dorsale de la nation.

Le fait que, à cause de l' «interprétation nationale », la décision d'utiliser la famine prit en Ukraine et dans le Kouban des traits tout à fait spécifiques est confirmé par les mesures qui étaient, du moins en partie, très différentes de celles adoptées à l'échelle de l'Union, à l'exception partielle des terres des Cosaques du Don.

Le 18 novembre, le Comité central ukrainien que Molotov et Kaganovič avaient contraint à l'obéissance, ordonna aux paysans de restituer les maigres avances en nature sur la nouvelle récolte qu'ils venaient de recevoir en paiement du travail accompli. La décision (il n'est pas difficile d'imaginer comment se traduisait en fait son application dans des villages où sévissait la faim) ouvrit la voie à la répression des fonctionnaires locaux qui avaient aidé les familles paysannes les plus affamées en distribuant leur blé. Des centaines de ces fonctionnaires furent fusillés, des milliers d'entre eux furent arrêtés souvent sous l'accusation de «populisme ». En même temps, en Ukraine et dans le Kouban, l'État introduisait des amendes en nature pour priver les paysans également de viande et de pommes de terre, mesure qui ne fut pas étendue à la Volga où - peut-être à l'exception de la République autonome allemande - Postyšev traita moins durement même les cadres locaux (cette punition moins cruelle causa toutefois un nombre très élevé de morts d'inanition). Certains secteurs du Caucase du Nord et d'Ukraine, où l'opposition à la collectivisation avait été plus forte, furent au contraire punis avec une cruauté encore plus grande : tous les biens qui s'y trouvaient, même les biens non agricoles, furent retirés des magasins locaux, cependant que dans certaines localités on procéda à la déportation vers le nord et vers l'est de la population tout entière.

La famine prit ainsi des formes et des dimensions de loin supérieures à celles qu'elle aurait eues si la nature avait suivi son cours. Bien que beaucoup moins intense et moins étendue que la famine de 1921-1922 en termes de sécheresse et de zones frappées (ainsi, la récolte de 1932, quoique très faible, fut même plus élevée que celle de 1945 où il n'y eut pas de morts massives imputables à la faim), la famine fit trois à quatre fois plus de victimes à la suite de décisions politiques qui visaient à sauver le régime de la crise dans laquelle il était tombé à cause de ces mêmes politiques, assurant ainsi la victoire de la « grande offensive » lancée quatre années auparavant.

De la conscience qu'en Ukraine et que dans le Kouban la question paysanne était aussi une question nationale découlait la nécessité de les affronter et de les « résoudre» toutes les deux d'un seul coup. Et pour s'assurer qu'une telle « solution» durerait dans le temps, on l'assortit de la décision d'éliminer les élites nationales et leurs politiques, soupçonnées, comme on le sait, de connivence avec les paysans.

Les 14 et 15 décembre 1932, le Politbjuro approuva deux décrets secrets qui bouleversaient dans la seule Ukraine les politiques officielles en matière de nationalité sanctionnées en 1923. On y lisait que l'indigénisation (korenazicija) déjà 
mentionnée, telle qu'elle avait été appliquée en Ukraine et dans le Kouban, loin d'avoir affaibli le sentiment national, l'avait au contraire aidé à se développer, en produisant des ennemis ayant la carte du parti en poche (rappelons que l'un des préceptes de la korenizacija était précisément le recrutement et la promotion des cadres locaux dans les structures du parti). Les paysans n'étaient donc pas seuls coupables de la crise dont ils partageaient la responsabilité avec la classe politique et intellectuelle ukrainienne.

Un tel raisonnement concernait aussi les programmes d'ukrainisation de la République russe. Plusieurs millions d'Ukrainiens qui, à la suite de choix pro-russes au moment du tracé des frontières entre les républiques au milieu des années 20 , se trouvaient vivre en RSFSR, furent ainsi privés du droit à l'instruction et à la presse dans leur propre langue et à une autonomie gouvernementale dont en revanche toutes les autres nationalités continuaient à jouir. Au moment du recensement de 1937, seuls trois millions de citoyens de la RSFSR se définirent comme Ukrainiens, contre 7,8 millions qui avaient fait ce choix en 1926 (mais une partie au moins de cette baisse peut être imputée à la promotion du Kazakhstan - république autonome de la RSFSR jusqu'à 1936 - devenu une république soviétique).

Quelques jours plus tard, le 19 décembre, des mesures semblables frappèrent la Biélorussie où, comme en Ukraine, la question paysanne coïncidait largement avec la question nationale et avait, pour cette raison, causé de sérieux problèmes déjà du temps de la guerre civile, même si c'était à une échelle infiniment moindre. En Biélorussie également, le parti fut accusé par la suite, au début du mois de mars suivant, de favoriser le nationalisme, et les cadres politiques locaux et l'intelligentsia nationale furent réprimés pour ce « crime », mais la répression fut moins brutale et on n'arriva pas, par exemple, au rejet explicite de la «biélorussisation ». On voyait ainsi confirmée la différence fondamentale dans les politiques nationales qui étaient beaucoup plus tolérantes en Asie Centrale ou en Sibérie qu'elles ne le furent dans les régions occidentales de l'URSS que Moscou considérait à juste titre comme beaucoup plus dangereuses ${ }^{34}$.

La nuit du 20 décembre, sur une proposition de Kaganovič, le Politbjuro ukrainien s'employa à réaliser de nouveaux objectifs dans le domaine des collectes de céréales. Neuf jours plus tard, on déclara que la précondition nécessaire pour atteindre de tels objectifs était la découverte et la saisie des « réserves familiales » ${ }^{35}$. Le 22 janvier 1933, tout de suite après l'arrivée de Postyšev, accompagné de centaines de cadres centraux, en qualité de nouveau plénipotentiaire de Moscou en Ukraine, Stalin et Molotov ordonnèrent à l'OGPU d'arrêter l'exode d'Ukraine et du Kouban des paysans à la recherche de nourriture. Le Comité central et le gouvernement, écrivaient-ils « sont convaincus qu'un exode comme celui de l'année passée a

34. Oleg Hlevnjuk m'a aimablement rappelé l'existence des décrets du Bureau politique: O sel'sko-hozjajstvennyh zagotovok $v$ Belorussii [Les stocks de céréales en Biélorussie], Politbjuro du 6 décembre 1932, procès-verbal n. 126, p. 1, RGASPI, fond 17, opis' 3, delo 912, list 8, 42-43 ; et $\mathrm{Ob}$ izvraščenii nacional'noj politiki $\mathrm{VKP}(\mathrm{b})$ v Belorussii [La déformation de la politique nationale du parti en Biélorussie], RGASPI, f. 17, op. 3, d. 917,1. 7.

35. Tragedija, p. 603-611. 
été organisé par les ennemis du pouvoir soviétique, par les socialistes-révolutionnaires et les agents polonais pour faire de l'agitation, 'en utilisant les paysans' contre les kolkhozes et, plus généralement, contre le pouvoir soviétique dans les territoires du nord. L'année passée les organes du parti, de l'État et de la police n'ont pas mis en lumière ce complot contre-révolutionnaire [...] Une répétition de cette erreur pendant l'année en cours serait intolérable ${ }^{36}$. Au cours du seul mois suivant, sur la base de ce décret, on arrêta au moins 220000 personnes, surtout des paysans affamés à la recherche de nourriture. On réexpédia 190000 d'entre eux crever de faim dans leurs villages.

Même les routes qui menaient aux villes ukrainiennes, incomparablement mieux approvisionnées que les campagnes, encore qu'elles fussent misérables ${ }^{37}$, furent entourées de barrages de police anti-paysans, alors que les villages étaient abandonnés à la mort. Ce que Kosior, encore secrétaire du parti ukrainien mais pour peu de temps, écrivit à Moscou le 15 mars confirme que la faim était utilisée pour apprendre aux paysans la soumission à l'État et à son maître : «l'insatisfaisante progression des ensemencements dans de nombreuses régions, déplorait-il, montre que la famine (golod) n'a pas encore enseigné la raison à bien des kolkhoziens » (c'est moi qui souligne) ${ }^{38}$.

Ces mesures furent accompagnées et suivies d'une vague de terreur anti-ukrainienne présentant déjà certains des traits qui marquèrent quelque temps après les « opérations de masse » de la Grande terreur de 1937-1938. Ainsi s'achevait par le suicide de leaders importants comme Skrypnyk et d'écrivains comme Hvyl'ovyj, et par la répression de milliers de ses cadres, l'expérience nationale-communiste suscitée par la guerre civile.

Par conséquent l'adoption du terme Holodomor semble légitime et même indispensable pour faire la distinction entre la famine générale de 1931-1933 et la famine ukrainienne après l'été de 1932. Malgré leurs liens étroits, les deux phénomènes furent en effet fondamentalement différents.

Il en va de même pour les conséquences de la famine, elles aussi en partie semblables et essentiellement différentes tout à la fois. Alors que, dans l'URSS tout entière, l'arme de la faim brisa la résistance paysanne ${ }^{39}$, en assurant la victoire d'un

\section{Tragedija, p. 635.}

37. Dans leurs rapports, les consuls italien et polonais de Kiev parlaient de centaines, et non de dizaines, de cas de morts d'inanition par jour dans les rues et dans les cours de la ville. Il s'agissait en général de paysans qui avaient réussi d'une manière ou d'une autre à gagner la ville en franchissant les cordons de police. Leurs corps étaient rapidement enlevés.

38. Voici le texte original : « to, čto golodovka ne naučila ešče očen’ mnogo kolhoznikov umurazumu, pokazyvaet neudovletvoritel'naja podgotovka k sevu kak raz v najbolee neblagopolučnyh rajonov » (in S. V. Kul’čyc’kyj, éd., Holodomor 1932-1933 rr., op. cit.).

39. Dès le 17 mai 1933, après avoir visité la région du Don, un inspecteur du Comité exécutif central de l'URSS signalait une légère augmentation du nombre de kolkhoziens au travail, en expliquant ce fait par leur désir de recevoir la nourriture que les autorités avaient commencé à distribuer sur la base des jours de travail effectif. Dans beaucoup de villages, ajoutait-il, la « conspiration du silence » avait été brisée : les paysans qui, encore quelques semaines auparavant, refusaient fût-ce même de parler avec les autorités, avaient commencé à intervenir aux réunions, en général pour demander du pain, contre la promesse de travailler avec zèle. Ainsi, de 
dictateur à l'égard duquel les gens éprouvaient une peur sans précédent, et autour duquel commença à se développer un nouveau culte, basé en grande partie sur cette peur ; en ouvrant la porte à la terreur de 1937-1938; en marquant un changement profond du mensonge officiel qui avait accompagné le régime soviétique depuis sa naissance ; en permettant, en vertu de la soumission de la république la plus importante, la transformation de fait de l'État fédéral soviétique en un empire despotique ; et enfin, en laissant un terrible héritage de douleur à de nombreuses familles qui ne furent même pas autorisées à en faire le deuil parce que la famine devint immédiatement peut-être le principal tabou du passé soviétique et que, d'après le nouveau dogme officiel, la vie était «devenue plus gaie » (il faut rappeler que Gorbačev aussi avait perdu dans la famine trois oncles paternels dont il a parlé ensuite dans ses mémoires, mais dont la mort, du moins en apparence, n'avait pas entaché son adhésion au régime) ${ }^{40}$, en Ukraine et au Kazakhstan la famine avait creusé beaucoup plus profondément.

Au Kazakhstan, la famine endommagea sérieusement les structures porteuses de la société traditionnelle. En Ukraine, tant le corps que la tête de la société nationale avaient été frappés, ralentissant et perturbant le processus d'édification nationale. Je pense, par exemple, que seul cet éclairage permet d'expliquer la présence incomparablement plus faible du mouvement national ukrainien dans la grande crise de 1941-1945, en comparaison de ce qui se produisit en 1914-1922 (la Galicie qui ne faisait pas partie de l'URSS en 1933 fut - ce qui n'est pas surprenant - 1'exception extraordinaire).

\section{Un génocide?}

Le nombre de leurs victimes fait des famines soviétiques de 1931-1933 un ensemble de phénomènes qui, dans le cadre de l'histoire européenne, peut être comparé uniquement aux crimes nazis ultérieurs. Le cours des événements en Ukraine et dans le Caucase du Nord, le lien qu'il eut tant avec l'interprétation que Stalin donna à la crise qu'avec les politiques qui en découlèrent, posent, de façon nouvelle, la question de leur nature : est-ce qu'il y eut aussi un génocide ukrainien ?

La réponse semble être non si nous pensons à une famine conçue par le régime ou — version encore plus indéfendable - par la Russie pour anéantir le peuple ukrainien. Et elle reste non si on adopte une définition restrictive du génocide comme une volonté prédéterminée d'exterminer tous les membres d'un groupe ethnique, religieux ou social, auquel cas seul l'Holocauste rentrerait dans cette catégorie.

la même façon et plus qu'en 1921-1922, la famine sauva le régime en brisant l'échine des paysans (in N. Werth, G. Moullec, Rapports secrets soviétiques, op. cit.). Le 11 juillet, un diplomate italien soutint la même thèse en se fondant sur les dires de plusieurs spécialistes agraires allemands revenus d'Ukraine et du Kouban (in A. Graziosi, Lettere da Kharkov..., op. cit., p. 152).

40. M. Gorbachev, Memoirs, New York, 1995, p. 27. 
De même la définition assez étroite de génocide adoptée par les Nations Unies en 1948 énumère cependant parmi les actes génocidaires possibles, outre le fait de «killing members of the group, and causing serious bodily or mental harm to members of the group », le fait de « deliberately inflicting on members of the group conditions of life calculated to bring about its physical destruction in whole or in part » (c'est moi qui souligne). Peu de temps auparavant, Raphael Lemkin, auquel nous devons ce terme, avait noté que, "généralement parlant, le génocide ne signifie pas forcément la destruction immédiate d'une nation... Par ce terme, on entend plutôt un plan coordonné de multiplication des actes visant à détruire les fondements essentiels de la vie de groupes nationaux... $»^{41}$.

Dans cette perspective, si nous réfléchissons aux différences importantes entre les taux de mortalité dans les diverses républiques ; si nous ajoutons aux millions de victimes ukrainiennes, celles du Kouban incluses, les millions d'Ukrainiens russifiés après décembre 1932, et les milliers de paysans en fuite qui connurent le même sort après avoir échappé aux barrages de police et s'être réfugiés dans la République russe ; si nous tenons compte du fait que nous avons affaire par conséquent à la perte de 20 à $25 \%$ de la population ethnique ukrainienne ; si nous nous remémorons que cette perte fut causée par la décision - un acte subjectif indubitablement - d'utiliser la famine dans le sens anti-ukrainien sur la base de l' «interprétation nationale » de la crise élaborée par Stalin dans la seconde moitié de 1932 ; si nous estimons que, sans cette décision, les victimes auraient été au maximum de l'ordre de centaines de mille, c'est-à-dire moins que pendant la famine de 1921-1922 ; et si finalement nous prenons en considération la destruction d'une grande partie de l'élite politique et intellectuelle de la république, des instituteurs de village aux leaders nationaux, alors la réponse à la question du génocide ukrainien ne peut qu'être positive.

Les événements compris entre la fin de 1932 et l'été 1933 permettent donc quelques conclusions :

1. Stalin et le régime contrôlé par lui et tenu en sujétion - mais certainement pas la Russie ou les Russes qui pâtirent eux aussi de la faim, même si ce fut à moindre échelle - appliquèrent consciemment, au sein d'une offensive tendant à faire céder les paysans, une politique anti-ukrainienne visant à l'extermination de masse et provoquant un génocide dans l'acception que je viens de donner au terme ${ }^{42}$, un génocide dont les cicatrices physiques et psychiques sont encore visibles aujourd'hui.

41. Yearbook of the United Nations, New York, 1948-1949, p. 959 ; R. Lemkin, Axis Rule in Occupied Europe, Washington, DC, 1944, p. 82. Voir J. O. Pohl, «Stalin's Genocide against 'Repressed People' », Journal of Genocide Research, 2, 2000, p. 267-293.

42. Comme N. Valentinov (Vol’skij) le souligna dans un article court mais pénétrant, « Tout est permis », Le Contrat social, X, 1966, p. 19-28 et 77-84, Stalin et Hitler ont fait partie du minuscule groupe d'un certain type terrible de révolutionnaires du $\mathrm{XX}^{\mathrm{e}}$ siècle européen, ceux pour lesquels justement tout était possible. 
2. Ce génocide est l'œuvre d'une famine qui, au début, n'avait pas été déclenchée artificiellement dans ce but, mais qui, une fois qu'elle eut fait son apparition comme conséquence «non programmée » des politiques du régime, fut manœuvrée volontairement à cette fin (il semble que la tragédie kazakhe, proportionnellement encore plus terrible, ait été «seulement» le résultat malencontreux de la dénomadisation et de l'indifférence absolue au sort des autochtones $^{43}$ ).

3. Ce génocide eut lieu dans un contexte dominé par la décision de Stalin de punir par la faim et par la terreur un certain nombre de groupes nationaux et ethnico-sociaux considérés comme dangereux ou potentiellement dangereux ${ }^{44}$. Ainsi que tous les indicateurs quantitatifs le montrent, la punition comme la terreur culminèrent, pour les raisons précédemment énumérées, en Ukraine où elles se transformèrent en un phénomène qualitativement différent.

4. Vues sous cet angle, les relations entre le Holodomor et les autres punitions répressives tragiques de 1932-1933 rappellent d'une certaine façon les rapports déjà mentionnés entre la répression nazie et l'Holocauste.

5. Toutefois, le Holodomor a été très différent de ce dernier. Il ne se proposait pas l'extermination de la nation ukrainienne tout entière, il ne reposa pas sur le meurtre direct des victimes, il fut motivé et élaboré théoriquement et poli-

43. La thèse du génocide a été soutenue également sur la base d'autres arguments. Kul’čyc'kyj, par exemple, a présenté aussi bien la famine à l'échelle de l'URSS que le Holodomor comme des génocides motivés idéologiquement, dans la mesure où ils résultaient de choix inspirés en 1929 par la conception de l'idéologie communiste alors courante parmi les dirigeants soviétiques. Le fait que derrière la « révolution stalinienne d'en haut », et donc derrière les politiques suscitées par la crise de 1931-1932, il y ait eu aussi des idéaux communistes - pour primitifs qu'ils aient été - semble incontestable. Et il est difficile de soutenir que Stalin a ignoré quelles pourraient être les conséquences de ces politiques. L'année 1921-1922 l'avait déjà démontré et, avant 1927, Stalin lui-même avait critiqué plusieurs fois la proposition de Trockij d'abandonner la NEP au profit de la collectivisation et de l'industrialisation accélérées (quoique de bien moindre envergure que celles qui furent promues par Stalin lui-même après 1928), en déclarant que ces mesures conduiraient à une crise dans les rapports avec les campagnes et provoqueraient une famine (terme employé par Stalin). Les hypothèses comme celles de Kul'čyc'kyj renferment donc plus qu'un noyau de vérité, mais je crois que Stalin, tout en sachant que l'offensive déclenchée en 1929 produirait une crise, n'imaginait pas alors les dimensions que celle-ci prendrait. En effet, à la fin de 1930 il était convaincu que le pire était passé et qu'il avait gagné la bataille avec les campagnes. Voici pourquoi ces positions, bien qu'elles soient en partie correctes et mettent en relief avec justesse le rôle de l'idéologie communiste et des croyances économiques erronées, sont faibles si on les utilise pour soutenir que la famine et le Holodomor furent des génocides.

44. Dans une lettre qu'il m'a adressée, Oleg Hlevnjuk a observé à juste titre que bien des politiques staliniennes présentaient des caractéristiques « génocidaires ». «Quel que fût le problème qui surgissait dans le pays, il était affronté par l'application de la violence directe à l'égard de groupes spécifiques de la population, et bien définis en termes socio-culturels, ou nationaux. » Ces groupes et le traitement qui leur fut infligé, depuis des mesures préventives jusqu'à la liquidation, varièrent au cours du temps en fonction de la situation intérieure et internationale et des convictions personnelles du despote. Ils comprenaient les Cosaques, les paysans, la vieille intelligentsia russe, et les intelligentsias nationales, les «nations ennemies » comme les Polonais et les Allemands d'abord, les Tchétchènes et les Juifs plus tard, etc. Par conséquent le Holodomor est posé sur cette toile de fond et ne peut être compris que dans ce contexte. 
tiquement - peut-on dire « rationnellement » $?^{45}$ — et non pas sur des bases ethniques et raciales (cette dernière différence est, au moins en partie, à l'origine des deux qui la précèdent).

6. Dans cette optique, l'Holocauste est exceptionnel, car il représente la forme la plus pure, et par conséquent qualitativement différente, de génocide imaginable. Il fait donc partie d'une catégorie indépendante, mais en même temps il représente le haut d'une pyramide à plusieurs étages dont les gradins sont constitués d'autant de tragédies. Celui du Holodomor se trouve proche de son sommet.

Si elle était exacte, comme je le crois, cette réponse affirmative aurait des conséquences morales et intellectuelles capitales sur notre image et sur notre interprétation du $\mathrm{XX}^{\mathrm{e}}$ siècle européen. Dans un autre essai, j'ai tenté d'indiquer quelques-unes de ces conséquences après avoir discuté les problèmes liés à l'impact de la «Grande famine », à moyen et long terme, sur l'histoire soviétique ${ }^{46}$. Je voudrais maintenant en rappeler seulement trois.

De quelle façon la conscience des modalités, de l'importance et des responsabilités de la famine influence-t-elle le jugement que nous sommes appelés à porter en tant qu'êtres humains avant même de le porter en tant qu'historiens - sur le système soviétique et sur la première génération de ses dirigeants, groupe dans lequel sont inclus les fonctionnaires qui exécutèrent leurs décisions, sans oublier pour autant ceux, nombreux, qui refusèrent courageusement d'appliquer et même boycottèrent les politiques de l'État, et furent sévèrement punis pour cela ?

À la lumière de 1932-1933, ce système ne nous apparaît-il pas, au moins en ce qui concerne une phase importante de son existence, bien plus comme un État violent et primitif, dirigé par un despote cruel, que comme un «totalitarisme» modernisateur, visant au nom de l'idéologie à conquérir et à remodeler les consciences de ses sujets?

Est-il possible de soutenir que, si à la racine du système soviétique, tel qu'il fut remodelé par Stalin, il y eut un crime d'une telle importance, alors son effondrement est en quelque sorte lié à ce péché originel, couvert par des décennies de mensonge, inavouable précisément pour cette raison. De ce point de vue, la « Grande famine » prit la forme d'un obstacle formidable à la survie, par renouvellement, d'un système qui ne pouvait dire la vérité sur son passé et fut balayé aussi par l'émergence de cette vérité, souvent grâce à des hommes qui voulaient le

45. On pourrait soutenir que les génocides pour des raisons raciales ou sur la base des théories du complot, selon lesquelles le futur d'une nation ou d'une « race » exige l'extermination d'un autre peuple, sont tout aussi « rationnels ». Après tout, la décision d'exterminer procède de ce qui pourrait passer pour un raisonnement. Je crois cependant qu'il y a là une différence importante, du moins dans le type de rationalité auquel nous faisons référence. Celle de Stalin était assez sophistiquée et impliquait l'utilisation de théories élaborées sur le processus d'édification nationale et étatique, sur le comportement paysan, sur les possibilités d'influencer la réalité, etc., qui plongeaient naturellement leurs racines dans le marxisme.

46. A. Graziosi, « The Great Famine of 1932-33: Consequences and Implications », à paraître dans Harvard Ukrainian Studies. 
réformer et le rendre plus humain, et commencèrent à le faire en cherchant à régler les comptes avec le passé, seulement pour découvrir qu'il y avait des comptes qui ne pouvaient pas être soldés ${ }^{47}$.

Ainsi se trouve soulevée de façon implicite la question, extrêmement intéressante, de l'évolution des «totalitarismes », une catégorie que je n'aime pas non plus parce qu'elle permet difficilement de tenir compte de cette évolution, indiscutable dans le cas soviétique (il suffit à ce propos de comparer Stalin non pas à Gorbačev, mais aussi à Hruščev et à Brežnev). D'après Burckhardt, « même un État fondé au début seulement sur le malheur des opprimés est contraint avec le temps à développer une espèce de droit et de vie civile, parce que les personnes civiles et justes s'en sont emparées petit à petit [...] et parce que [l'État peut] prouver sa vitalité seulement en transformant la violence en force. $\gg^{48}$

Se peut-il, si la paix prévaut pendant une période suffisamment longue, que la progression, si ce n'est le triomphe de cette évolution, soit vraiment réalisable même quand l'histoire du système qui évolue est marquée par un génocide ? Si c'était vrai, l'histoire soviétique ne serait pas seulement cette parabole morale extraordinaire qu'elle est, mais aussi un signe d'espoir en termes bien plus généraux.

(traduit de l'italien par Dominique Négrel)

\section{Università degli studi di Napoli Federico II}

andrea.graziosi@unina.it

47. Bien entendu, je ne soutiens pas que ceci ait été la raison de l'effondrement soviétique. Pourtant la nature inexpiable de son passé compliqua certainement la vie d'un système qui était en train de suffoquer lentement sous le poids de ses contradictions démographiques, économiques et nationales et qui fut anéanti finalement par la tentative de lui trouver une nouvelle vie grâce à une grande réforme.

48. J. Burckhardt, Considérations sur l'histoire universelle, Paris, 2001 (édition italienne, Florence, 1965, p. 35 sq). 